\title{
The distribution of maser stars in the inner Milky Way: the effect of a weak, rotating bar
}

\author{
H. J. Habing, M. N. Sevenster, M. Messineo, G. van de Ven, and K. Kuijken
}

(The ROTBAR consortium)

\author{
Leiden Observatory, PO Box 9513, 2300 RA Leiden, The Netherlands \\ e-mail: habing@strw.leidenuniv.nl
}

Received 5 November 2005 / Accepted 26 July 2006

ABSTRACT

\begin{abstract}
We derive the distribution of maser stars in the inner Milky Way based on an analysis of diagrams of longitude versus line-of-sightvelocity (=lV-diagrams) for two samples of maser stars: $771 \mathrm{OH} / \mathrm{IR}$ stars and $363 \mathrm{SiO}$-maser stars. The stars are all close to the plane of the Milky Way and have longitudes from $-45^{\circ}$ to $+45^{\circ}$.

The two $l V$-diagrams are compared qualitatively and found to be very similar. They also compare well with the $l V$-diagram of interstellar $\mathrm{CO}$, but there are significant differences in detail between the stellar $l V$-diagrams and that of the ISM.

Based on the qualitative discussion we divide the $l V$-diagrams into seven areas. In each area we count the number of stars as observed and compare these numbers with those predicted by an assumed set of orbits in a galactic potential. This potential is axially symmetric but a weak rotating bar has been added. We conclude that the maser stars move on almost circular orbits outside of about $3.5 \mathrm{kpc}$, but that the orbits become more and more elongated when one goes deep inside our MW. We find a strong effect of the Corotation $(=\mathrm{CR})$ resonance at $3.3 \mathrm{kpc}$, we see a small but noticeable effect of the Outer Lindblad Resonance at $5 \mathrm{kpc}$ and no effect of the Inner Lindblad Resonance (=ILR) at $r=0.8 \mathrm{kpc}$.

We find a set of 6 groups of orbits that together predict counts in agreement with the counts of stars observed. We then calculate the trajectory of each orbit and so find the distribution of the maser stars in the plane of the MWG. This distribution has two new (but not unexpected) features. The first is a bar-like distribution within $2 \mathrm{kpc}$ from the GC outlined. These orbits explain the high-velocity stars near $l=0^{\circ}$ in the forbidden and the permitted quadrants. The second feature are two "croissant"-like voids in the distribution close to the $\mathrm{CR}$ radius $(3.3 \mathrm{kpc})$. These voids are the consequence of the presence of the co-rotation resonance. We find excellent agreement with an earlier reconstruction by Sevenster (1999) based on partially the same data but on a completely different analysis.
\end{abstract}

Key words. Galaxy: structure - stars: AGB and post-AGB - Galaxy: center

\section{Introduction}

We cannot see into the inner parts of our Milky Way Galaxy (=MWG) close to the galactic plane because of the extinction of light by dust particles in intervening interstellar clouds. Radiation with a wavelength of a few microns and longer is not affected by the dust and that makes it possible to detect and to study the objects in the inner Galaxy. In the 1950's, under the leadership of J.H. Oort, the distribution of the interstellar matter (=ISM) throughout the MWG was derived in an elegant and convincing manner using the results of a survey of the $21 \mathrm{~cm}$ line of HI. The success was not complete, however: about one third of the galactic plane map was blank: in directions within $30^{\circ}$ from the galactic center $(=\mathrm{GC})$ and galactic anticenter the kinematics of the gas could not be used to find the distance to the gas velocities and in addition the gas motions in the GC direction are complex and were not understood at that time. The problem is apparently solved by the introduction of a weak, rotating bar (see e.g. Bissantz et al. 2003). The $21 \mathrm{~cm} \mathrm{HI} \mathrm{line} \mathrm{data}$ have been complemented in the 1970's by surveys of the lowest rotational transition of $\mathrm{CO}$ at $115 \mathrm{GHz}(\lambda=2.6 \mathrm{~mm})$, a molecule that acts as substitute for molecular hydrogen. A very conspicuous feature is the (almost complete) absence of ISM inside the so-called "molecular ring" with a radius of about $3 \mathrm{kpc}$, except of the gas within a few hundred parsec of SgrA*. All these results are well known and have been described in text bookse.g. Binney \& Merrifield (1998) (hereafter BM).

In 1968 Wilson and Barrett anounced the detection of a red giant as the source of a strong emission line of the hydroxyl radical $(\mathrm{OH})$ at $1612 \mathrm{MHz}(18 \mathrm{~cm})$. The strength of the line by itself and in comparison to other lines from the same multiplet indicated that the line had been amplified by a maser process in the circumstellar environment of this star. Quickly thereafter Elldér et al. (1969) announced the discovery of several similar maser sources in a systematic survey of a small part of the Milky Way in Cygnus. These detections suggested that a "blind" survey at $1612 \mathrm{MHz}$ of a large fraction of the Milky Way might find enough masers to derive the distribution of these stars in the inner MWG, similar to what had been achieved for the ISM through the $21 \mathrm{~cm}$ line. In the following years two more maseramplified molecular lines were detected: $\mathrm{H}_{2} \mathrm{O}$ (Knowles et al. 1969) and $\mathrm{SiO}$ (Kaifu et al. 1975).

The suggestion of a larger, blind $1612 \mathrm{MHz}$ survey led to two searches for maser stars along the northern Milky Way (Bowers 1978a,b; Baud et al. 1981a). Some 200 stars were detected and from the observed relation between their longitude and latitude coordinate and their line-of-sight velocity ${ }^{1}$ it

\footnotetext{
1 We will use the word line-of-sight velocity instead of the more common term radial velocity because the latter will be reserved for stellar motions to and from the GC.
} 
appeared that the stars form a thin, rotating disk with low density (a "hole") within about $3 \mathrm{kpc}$ from the GC, much like the hole in the ISM. An unexpected result was the discovery of a star with a high negative velocity $\left(-341 \mathrm{~km} \mathrm{~s}^{-1}\right)$ at a positive longitude $(l, b)=\left(0.3^{\circ},-0.2^{\circ}\right)$ (Baud et al. 1975). More highvelocity stars were discovered later. They play an important role in this paper.

This paper deals with a renewed search for the galactic distribution of maser stars, using sources detected in two recent surveys, one in the $\mathrm{OH} 1612 \mathrm{MHz}$ line, the other in the $86 \mathrm{GHz}$ line of $\mathrm{SiO}$. The data obtained in these surveys have already been analyzed into quite some detail- as we will discuss below. Nevertheless we felt that further analysis might still yield some interesting results- hence this paper.

The paper is structured as follows. (1) We discuss the astrophysical properties of the maser star population. (2) A summary is given of what is already known about the relation between maser stars and the bar of our MWG. (3) The two diagrams of longitude versus line-of-sight velocity (=lV-diagrams) of the maser stars are compared qualitatively one with another and then with that of interstellar CO. (4) After adopting a galactic potential we calculate possible orbits for the maser stars in the galactic plane and their projection on the $l V$-diagram. (5) Using the $l V$ diagram of the stars as a guide we select the orbits in the galactic plane that the maser stars are likely to follow. (6) Using these orbits we derive the spatial distribution of the maser stars in the inner MWG.

\section{Physical properties of maser stars}

What stars show maser emission? An answer may be found in the reviews edited by Habing \& Olofsson (2003). When stars with a mass below 6 or $7 \mathcal{M}_{\odot}$ turn off the main sequence they will quickly go through a number of different appearances. In the last phase in which nuclear burning is still significant, the Asymptotic Giant Branch phase (=AGB), the star is very luminous, very red and it loses mass at a high rate but at a low velocity. The out-flowing matter is seen as a cold circumstellar envelope and it is in such envelopes that the $\mathrm{OH}, \mathrm{H}_{2} \mathrm{O}$ and $\mathrm{SiO}$ masers are produced. The AGB-phase is short-lived; perhaps it lasts as short as $50000 \mathrm{yr}$ : maser stars are rare, but they represent ordinary stars of low and intermediate mass. Model calculations predict that the luminosity of an AGB star is tightly correlated with the age and the metallicity of the star. It is still too early to exploit this correlation but one important conclusion can already be drawn: maser stars represent objects of an age $(0.2$ to 2 Gyr) that is intermediate between the age of RR Lyrae and globular clusters (10 Gyr) and that of OB-stars and open clusters $(\leq 0.2$ Gyr).

Almost all OH/IR stars in the Milky Way have been found within $50^{\circ}$ from the GC in spite of deep searches farther awaye.g. te Lintel Hekkert et al. (1991): there are very few maser stars known outside the solar circle, and those few are probably of high mass. This rareness of maser stars in the outer MWG can be explained by a lower metallicity: when stars of low metallicity reach the AGB phase they develop into carbon stars and there are not enough free $\mathrm{O}$-atoms left in the circumstellar envelope to form the $\mathrm{OH}$ radical or the $\mathrm{SiO}$ molecule in sufficient numbers. If a maser is seen only when the stellar metallicity is high enough, galactic metallicity introduces a bias in the samples of detected maser stars.

Concerning terminology: the $\mathrm{OH}$-maser stars discussed here have all been detected in the $1612 \mathrm{MHz}$ line, and will hence be called "OH/IR stars". Stars detected because of their SiO maser emission will be called "SiO-maser stars".

\section{Existing surveys for maser stars; previously derived conclusions}

We have at our disposal a sample of 1134 maser stars $(771 \mathrm{OH}$, $363 \mathrm{SiO}$ masers), all at low galactic latitudes $\left(|b| \leq 3.5^{\circ}\right)$ and in the inner MWG $\left(|l| \leq 45^{\circ}\right)$. The OH/IR sample contains all $\mathrm{OH} / \mathrm{IR}$ maser stars with a peak flux density above $0.5 \mathrm{Jy}$ in the area specified and with a line-of-sight velocity within $300 \mathrm{~km} \mathrm{~s}^{-1}$ from zero velocity in the Local Standard of Rest; see Sevenster et al. (1997a,b, 2001). The SiO maser sample has been obtained by pointing the telescope at infrared objects from the ISOGAL survey (see Messineo et al. 2002). Not all candidate infrared objects have been observed and the longitude distribution may not be representative; all infrared objects were within $1^{\circ}$ from the galactic plane. The $\mathrm{OH}$ - and the $\mathrm{SiO}$-. survey had a sensitivity limit low enough to find stars beyond the GC.

We limited ourselves to these two surveys because we had all the data readily available and because we understood the selection criteria and thus the completeness of the surveys. We therefore did not include the large sample of SiO-maser stars detected by Japanese astronomers, e.g. Deguchi et al. (2004b) who used the IRAS catalogue as the source of candidate stars, and this catalog is quite incomplete near the galactic plane. For the same reason we did not use the earlier OH/IR survey by te Lintel Hekkert et al. (1991). One other limitation concerns the immediate surroundings of the GC, roughly the area within $1.5^{\circ}$ from Sgr A*. Results from deeper $\mathrm{OH}$ surveys in this area are available (Lindqvist et al. 1992a,b; and Sjouwerman et al. 1998) but as we believe that the structure in this area has its own difficulties that are not of importance for the remainder of the MWG we did not discuss these surveys and leave those for a future paper.

Consider now the conclusions already reached concerning the distribution in position and in velocity of the maser stars as far as these are relevant for our present study.

1. Sevenster et al. (2000) calculated orbits of stars in a threedimensional MWG model under the assumption that there are two integrals of motion, the energy, $E$, and the angular momentum around the $z$-axis, $J$. A quite significant conclusion follows from their Fig. 6: the distribution of the angular momenta changes gradually at energies $E$ corresponding to circular orbits with a radius $r=2.0 \mathrm{kpc}$. At higher energies (i.e. farther outside in the disk) the stars move all on almostcircular orbits and in the clock-wise direction as seen from the galactic north pole. At lower energies and thus deeper inside the MWG the distribution of the angular momenta becomes flat: for a given energy all angular momenta have the same probability and there are as many circular orbits in the anti-clock-wise direction as in the clock-wise direction.

2. If in a galaxy with a rotating bar the density distribution is described as a function of polar coordinates $(r, \phi)$ the dependence on time must be such that $\phi=\phi_{0}-\Omega t$. Tremaine \& Weinberg (1984) have shown that as a consequence of this symmetry the value of $\Omega$ can be derived from the measurement of line-of-sight velocities in an edge-on galaxy. Using a data base of $\mathrm{OH} / \mathrm{IR}$ stars almost the same as ours Debattista et al. (2001) calculated the pattern speed of a bar in the inner MWG and obtained the value $\Omega=-59 \pm 5 \mathrm{~km} \mathrm{~s}^{-1} \mathrm{kpc}^{-1}$. The tangential velocity of the maser stars thus contains a systematic component that is proportional to the distance of the star 
to the GC. This is a strong argument in favor of the statement that the $\mathrm{OH} / \mathrm{IR}$ stars are part of the galactic bar.

3. Sevenster (1999b) analyzed a sample of $509 \mathrm{OH} / \mathrm{IR}$ stars in the longitude range from $l=+10^{\circ}$ down to $l=-45^{\circ}$. She concludes: "Unequivocal morphological evidence is presented for the existence of a central Bar...". Corotation (CR) is at $3.5 \mathrm{kpc}$, the inner Lindblad resonance (ILR) is between 1 and $1.5 \mathrm{kpc}$ and the inner ultra-harmonic radius is at $2.5 \mathrm{kpc}$.

4. Sevenster et al. (1999) have compared several $N$-body simulations of the MWG with the OH/IR stars data obtained with the ATCA. In these simulations a bar is formed spontaneously. Good agreement between predictions and observations is found for a viewing angle of $44^{\circ}, \mathrm{CR}$ at $4.5 \mathrm{kpc}$.

5. In the same paper as in item 3 Sevenster points out that the distribution of the $\mathrm{OH} / \mathrm{IR}$ stars over positive and negative longitudes are slightly different and that this difference has to be attributed to the presence of the bar. We have now a larger data base and our conclusion confirms the one previous; see the next subsection.

We conclude that all these items, except 1 , argue that the maser stars are part of the bar of the MWG.

\subsection{Differences between the distribution at positive and at negative longitudes}

For a long time, the interpretation of asymmetries in the integrated-light distribution observed toward the inner Galaxy was limited by the degeneracy of the deprojection. In external galaxies, edge-on bars do not show up as asymmetries in the morphology, so asymmetries have to be interpreted as $m=1$ distortions (or odd-numbered in general). In the Galaxy, both $m=1$ and $m=2$ non-axisymmetries would result in an asymmetric surface morphology. Most observations before COBE (Weiland et al. 1994; Dwek et al. 1995) showed asymmetries that could not be unambiguously attributed to an $m=2$ distortion.

The COBE observations themselves showed a surfacedensity distribution with a clear hint of the Bar. The image is, however, still limited by extinction. As explained by Blitz \& Spergel (1991), when observing a tracer population that can be sampled out to $d>12 \mathrm{kpc}$, so that the bar is fully included, a signature would be seen that would point unambiguously toward the existence of a bar. This signature was first seen in a sample of $\mathrm{OH} / \mathrm{IR}$ stars (Sevenster 1999). With the extended sample of $\mathrm{OH} / \mathrm{IR}$ stars, we now repeat the test.

Figure 1 shows this signature. After arranging all $\mathrm{OH} / \mathrm{IR}$ stars according to their absolute longitude we show $N=\Sigma l_{i} /\left|l_{i}\right|$. If the distribution were axisymmetric $N$ would always remain close to 0 . This is clearly not the case. Out to about $|l|=10^{\circ}$ there is an overdensity at negative longitudes. Between $|l|=10^{\circ}$ and $30^{\circ}$ there is a strong overdensity at positive longitudes. At higher longitudes, the curve approximates a constant value, indicating a certain lopsidedness in the distribution of stars in our MWG. For a more elaborate discussion of this test, see Sevenster (1999). An important conclusion for this paper is that the sample of $\mathrm{OH} / \mathrm{IR}$ stars does sample the entire Bar, both the near and the far end. It provides a good sample for kinematical analysis of bar orbits, especially with the accurate line-of-sight velocities.

The same asymmetric distribution of stars has been found by Benjamin et al. (2005) in a much larger sample of red giants detected at mid-infrared wavelengths with the SPITZER satellite; the authors also argue for the existence of the bar.

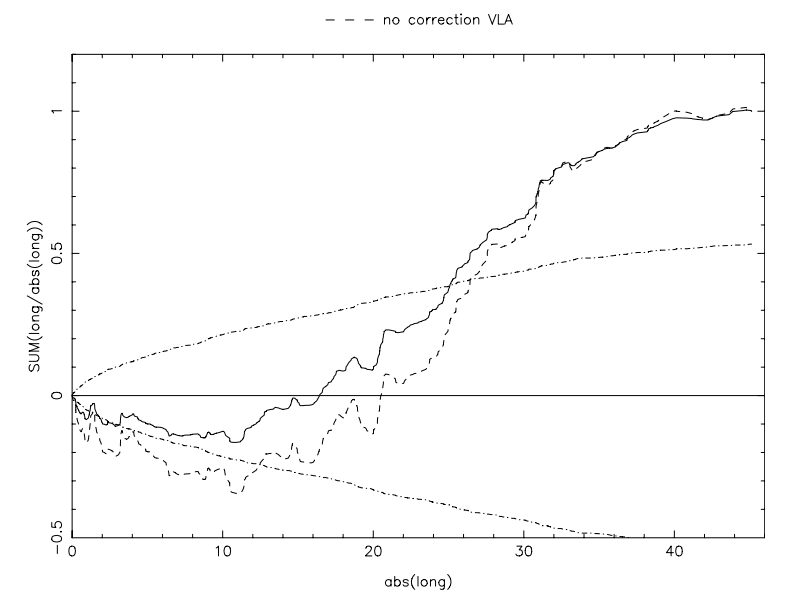

Fig. 1. The cumulative distribution of the sign of the longitude, as a function of absolute longitude. The solid curve indicates the distribution when correcting for the lower completeness of the VLA section of the survey (see Sevenster et al. 2001). The dashed curve indicates the distribution when no correction is applied, i.e. when effectively every star in the sample counts as 1 . A decreasing sum indicates an overdensity at negative longitudes, an increasing sum indicates an overdensity at positive longitudes. Horizontal sections in the curve indicate a symmetrical distribution of stars with respect to $l=0$. The dash-dotted curves give the two-sided $95 \%$ interval assuming the stars are drawn from a binomial distribution. Only OH/IR stars (614 in total) with outflow velocities between 2 and $20 \mathrm{~km} \mathrm{~s}^{-1}$ are included, to ensure a homogeneous population of stars that traces the Bar.

\subsection{Foreground extinction and distance from the Sun}

Stellar masers are formed in the dust-rich envelopes of AGB stars. In $\mathrm{OH}$ masers these envelopes are optically thick to wavelengths as large as $10 \mu \mathrm{m}$. SiO masers form in thinner envelope and if near-IR photometry is available one can derive $A_{\mathrm{K}}$, the sum of interstellar and circumstellar extinction of each maser star. As the maser stars are variables one needs simultaneously measured magnitudes. Existing surveys provide this information: $I-, J$ - and $K$-magnitudes by DENIS and $J$-, $H$ - and $K$-magnitudes by 2MASS. In all fields the maser star is (one of) the brightest sources and can easily be identified (Messineo et al. 2004, 2005). When we separate groups of maser stars based on their value of $A_{\mathrm{K}}$ we see systematic differences between their $l V$-diagrams; see Fig. 2 . These differences we attribute to differences in distance: larger values of $A_{\mathrm{K}}$ imply a larger distance. The few stars at longitudes larger than $20^{\circ}$ and with medium or large extinction may be located behind dense clouds. The systematic change in character between the three diagrams is in agreement with the model that we will develop later in this paper.

\section{IV-diagrams of maser stars and of IS gas: a qualitative comparison}

We will compare the two $l V$-diagrams of the maser stars with each other and with the $l V$-diagram of interstellar CO. The $l V$-diagrams are divided into four quadrants in the usual way, see the upper diagrams in Fig. 3. Q2 and Q4 are the well-known "forbidden quadrants" of circular galactic rotation. Q1 and Q3 will be called the "permitted quadrants". 


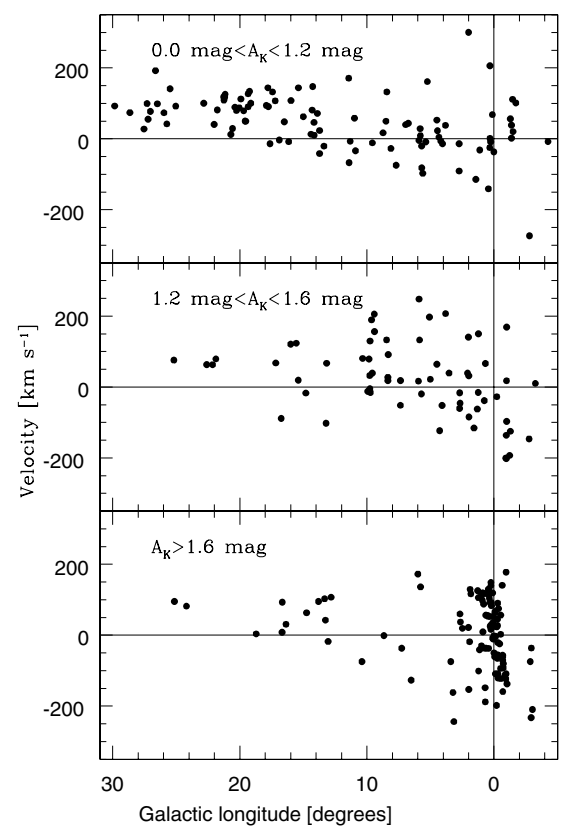

Fig. 2. $l V$-diagrams of different samples of SiO-maser stars; the samples have been selected using an estimate of the amount of the foreground extinction.

\subsection{Comparing the IV-diagrams of the maser stars}

The $l V$-diagram of the $\mathrm{OH} / \mathrm{IR}$ stars is the upper left diagram in Fig. 3 and that of the SiO-maser stars is the upper right diagram. The two samples of maser stars have very similar distributions:

1. The $v_{\text {los }}=0$ line is an approximate lower limit for $v_{\text {los }}$ at positive longitudes and an upper limit at negative longitudes; there are a few stars outside of this approximate limit.

2. The curve $v_{\text {los }}=200(1-\sin l) \mathrm{km} \mathrm{s}^{-1}$ is an upper limit in $\mathrm{Q} 1$ and $v_{\text {los }}=-200(1+\sin l) \mathrm{km} \mathrm{s}^{-1}$ a lower limit in Q3 for most of the stars. The longitude distribution of the $\mathrm{SiO}$ maser stars is not representative for the true distribution, but the distribution in $v_{\text {los }}$ is representative.

3. For $25^{\circ} \geq l \geq-25^{\circ}$ the curve $v_{\mathrm{los}}=284 \sin l \mathrm{~km} \mathrm{~s}^{-1}$ separates a region of lower density of stars from one of higher density.

4. Close to $l=0^{\circ}$ there is a significant number of stars with $\left|v_{\text {los }}\right|>200 \mathrm{~km} \mathrm{~s}^{-1}$.

A clear difference between the two $l V$-diagrams is the very tight correlation between $l$ and $v_{\text {los }}$ in the $\mathrm{SiO}$-maser stars near $l=0^{\circ}$; in the $\mathrm{OH} / \mathrm{IR}$ diagram such a sample is not present- although a trace of it may be seen by a favorably inclined reader. The lack of a similar set of $\mathrm{OH} / \mathrm{IR}$ stars is only apparent; in fact, in an earlier, deeper VLA study of the OH/IR stars in a small region around the GC (Lindqvist et al. 1992a,b) a sample of OH/IR stars was found with the same narrow correlation between $l$ and $V$. The correlation may be explained by solid body rotation caused by a constant density in the mass distribution inside $150 \mathrm{pc}$ from the GC (Lindqvist et al. 1992b), but another interpretation of the same data is certainly possible (Winnberg 2003).

\subsection{The IV-diagram of interstellar CO}

In the lower left corner of Fig. 3 we show the $\mathrm{CO} l V$-diagram by Dame et al. (2001).

1. The CO emission is strongly confined to the galactic plane, much more so than the maser stars.
2. The CO emission is (almost completely) limited to the permitted quadrants Q1 and Q3 and by the two curves defined by $v_{\text {los }}<200(1-\sin l) \mathrm{km} \mathrm{s}^{-1}$ for $l>0^{\circ}$ and by $v_{\text {los }}>$ $-200(1+\sin l) \mathrm{km} \mathrm{s}^{-1}$ for $l<0^{\circ}$. When the gas follows circular orbits these curves indicate the maximum $v_{\mathrm{los}}$ in the direction $l$.

3. There is some emission in the forbidden quadrants Q2 and Q4. (i) Close to $(l, V)=(0,0)$ a weak emission reaches measurable intensity in Q2; its border is given by a straight line through $(l, V)=(0,-57)$ and $(l, V)=(15,0)$. In $\mathrm{Q} 4$ the same is true; this time the border line runs through $(l, V)=(0,25)$ and $(l, V)=(-15,0)$. (ii) There is weak emission in the forbidden quadrants farther away from the center; this emission is almost certainly from gas outside of the solar circle at the far end of the MWG.

4. A curve defined by $v_{\text {los }}=280 \sin l \mathrm{~km} \mathrm{~s}^{-1}$, separates a region of high intensity and one of low intensity as well as in Q1 as in Q3; this is true both at positive and at negative longitudes in the $l V$-diagram of the $\mathrm{OH} / \mathrm{IR}$ stars and of the $\mathrm{SiO}$-maser stars (Fig. 3). This curve is the outer limit of the large and well-known hole in the ISM distribution inside of the molecular ring. If the ISM moves in perfect circles around the GC at a constant speed $v_{\text {circ }}$ the factor $280.4 \mathrm{~km} \mathrm{~s}^{-1}$ is fixed by the radius of the hole: $280.4=v_{\mathrm{o}}\left(r_{\mathrm{GC}} / r_{\text {hole }}-1\right)$ and because we use (see below) $r_{\mathrm{GC}}=8.0 \mathrm{kpc}$ and $v_{\mathrm{o}}=200 \mathrm{~km} \mathrm{~s}^{-1}$ we find $r_{\text {hole }}=3.3 \mathrm{kpc}$.

5. Two straight lines defined by $l= \pm 6^{\circ}$ limit the region of very high velocities around $l=0^{\circ}$. The most extreme velocity is $250 \mathrm{~km} \mathrm{~s}^{-1}$ in Q1 and $-250 \mathrm{~km} \mathrm{~s}^{-1}$ in Q3. High velocities are not found in the forbidden quadrants Q2 and Q4.

\subsection{Comparing the stellar and the ISM IV-diagrams}

The comparison of the two maser star $l V$-diagrams with each other shows these to be very similar. This is almost, but not quite the case when one compares the maser star $l V$-diagrams with that of interstellar CO.

1. The velocities of the stars are limited by the same curves and straight lines as the velocities of the gas but a small number of stars have wandered outside these curves.

2. There is quite some "trespassing" by the maser stars into the two forbidden quadrants Q2 and Q4, much more than shown by the ISM.

3. Our sample of $\mathrm{OH} / \mathrm{IR}$ stars contains 28 stars in the strip between $l=6^{\circ}$ and $l=-6^{\circ}$ with a velocity $\left|v_{\text {los }}\right| \geq 200 \mathrm{~km} \mathrm{~s}^{-1}$ and four $\mathrm{SiO}$-maser stars. Nineteen $\mathrm{OH} / \mathrm{IR}$ stars have "permitted" velocities (i.e. they are found in Q1 and Q3) and nine have "forbidden" velocities (i.e. they are found in Q2 and Q4); there is no high-velocity gas at forbidden velocities.

4. The curve $v_{\text {los }}=280 \sin l \mathrm{~km} \mathrm{~s}^{-1}$ separates a region of high source density and one of low source density in both Q1 and Q3.

\subsection{Conclusions based on our qualitative comparison}

The stellar $l V$-diagrams show a small but significant number of stars outside the limits strictly observed by the CO. We attribute this to the slow but continuous diffusion of stars in velocity space: stars accumulate perturbations of their velocity by incidental encounters, whereas the kinematics of the gas is dominated by dissipative forces. 

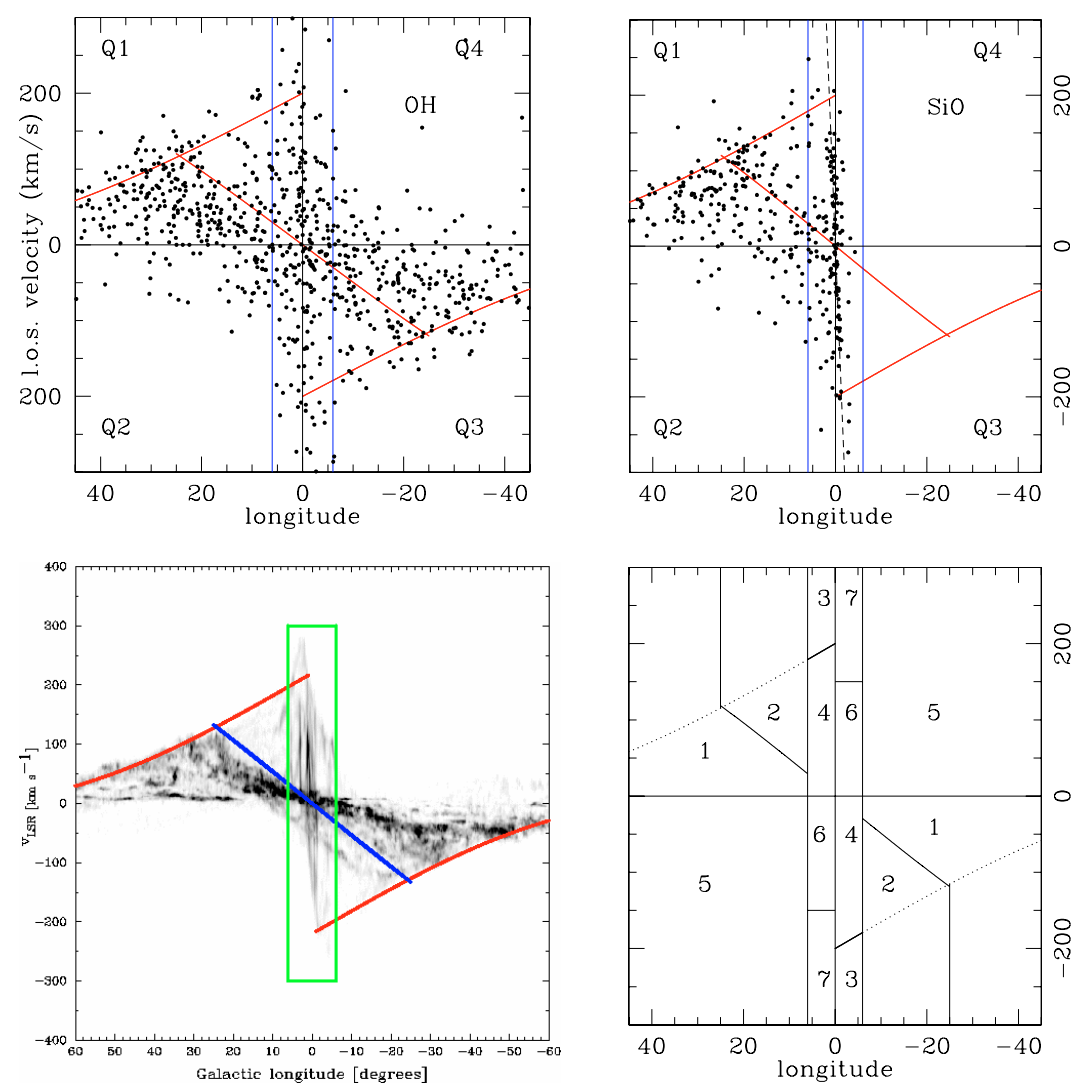

Fig. 3. The two diagrams at the top show the distribution of longitude and l.o.s. velocity of OH/IR stars (left) and SiO maser stars (right). The meaning of the red curves in these two diagrams is explained in the text. The dashed line in the $\mathrm{SiO}$ diagram is the line of correlation between longitudes and velocities of stars very close to the galactic center: $V=150 l \mathrm{~km} \mathrm{~s}^{-1}$ where $l$ is in degrees. The diagram of the lower left shows the diagram for the interstellar CO emission (Dame et al. 2001). The diagram at the lower right shows the limits of the areas defined in the text. The dotted curves are porous in the sense that they are a limit to most stars but not to all.

All in all we conclude that $l V$-diagrams of the maser stars agree with those of the ISM in their major features. The agreement thus implies that stars and gas have a very similar distribution in space and in velocity.

Our next step is to analyze the two stellar $l V$-diagrams by a quantitative, statistical method. Based on our qualitative comparison we divide the $l V$-diagrams into seven areas- see the diagram on the lower right in Fig. 3. We count the OH/IR stars in each area and compare the count to what we predict with a simple galactic potential to which a weak rotating bar is added. Each area has the same weight although different areas contain different numbers of stars. For example the small number of stars in area 7 carry the same weight as the much larger number of stars in area 1.

\section{A quantitative analysis of the IV-diagrams of the stars}

Our quantitative analysis of the stellar $l V$-diagrams is based on the orbits of stars calculated in a simple two-dimensional gravitational potential that contains a weak, rotating bar. From this potential we generate a library of orbits representative for our problem. Each orbit defines a probability distribution in the $l V$-diagrams. By taking linear combinations of orbits we predict numbers of stars in several areas in the $l V$-diagrams. We then use a $\chi^{2}$-method to find the best agreement between predictions and observations.

\subsection{Calculation of orbits; equations of motion}

We start from the equations given in the monograph by Binney \& Tremaine (1987, hereafter BT). We adopt a coordinate system corotating with the bar and with its origin at the center of the MWG. We start from the equation

$\ddot{\boldsymbol{r}}=-\nabla \Phi-2\left(\boldsymbol{\Omega}_{\mathrm{b}} \times \dot{\boldsymbol{r}}\right)$

and obtain as equations of motion:

$\frac{\mathrm{d}^{2} x}{\mathrm{~d} t^{2}}=-\frac{\partial \Phi}{\partial x}+\Omega^{2} x+2 \Omega \frac{\mathrm{d} y}{\mathrm{~d} t}$

and

$\frac{\mathrm{d}^{2} y}{\mathrm{~d} t^{2}}=-\frac{\partial \Phi}{\partial y}+\Omega^{2} y-2 \Omega \frac{\mathrm{d} x}{\mathrm{~d} t}$

In the right-hand side of Eqs. (2) and (3) the first term is the gravitational acceleration in the direction of the $x$-, resp. $y$-axis; the second term is the centrifugal acceleration and the third term is the Coriolis acceleration. The Coriolis acceleration introduces a dependence of the acceleration in the $x$-direction on the velocity in the $y$-direction and vice versa.

We solved Eqs. (2) and (3) numerically with the fourth order Runge-Kutta algorithm described in "Numerical Recipes". We tested the code successfully by calculating the same circular orbit in frames rotating at different speeds including speed zero. The calculation of the Jacobian energy (see BT) was another test; this energy was constant to within 1 in 1000 . 
The next step is to calculate the longitude $l$ of the star and its line-of-sight velocity $v_{\text {los }}$ at a given moment in time $t$. Let the cartesian coordinates of the star be $(x, y)$ and its polar coordiates $(r, \phi)$; the corresponding coordinates of the Sun are $\left(x_{\odot}, y_{\odot}\right)$ and $\left(r_{\odot}, \phi_{\odot}\right)$. The coordinate $\phi_{\odot}$ is the angle between the long axis of the bar and the Galactic Center - Sun direction. The parameter $\phi_{\odot}$ is one of the important free parameters of our problem. Consider the triangle of the Sun (symbol Z), the star (symbol S) and the galactic center (symbol C). The angle $\mathrm{SCZ}$ will be called $\beta$; its value is $\beta=\phi-\phi_{\odot}$. The distance $d$ from the Sun to the star is given by $d^{2}=r^{2}+r_{\odot}^{2}-2 r r_{\odot} \cos \beta$. The longitude $l$ is derived from the equation $\sin (-l) / r=\sin \beta / d$; the minus sign in front of $l$ takes into account the definition of longitude in the counterclock direction when viewed from the galactic north pole. For the calculation of $v_{\text {los }}$ we have to add the velocity of rotation of the frame and thus obtain the velocity of the star in an inertial frame that coincides with the rotating frame at the time $t:\left(\dot{x}_{\text {in }}, \dot{y}_{\text {in }}\right)=(\dot{x}-r \Omega \sin \phi, \dot{y}+r \Omega \cos \phi)$. The projection of the velocity of a given star on the line-of-sight equals $v_{\text {los }, 1}=\dot{x}_{\text {in }}\left(x-x_{\odot}\right) / d+\dot{y}_{\text {in }}\left(y-y_{\odot}\right) / d$. The projection of solar velocity on the line-of-sight equals $v_{\mathrm{los}, 2}=v_{\odot} \sin l$ and $v_{\mathrm{los}}$ is the sum of the two projections: $v_{\mathrm{los}}=v_{\mathrm{los}, 1}+v_{\mathrm{los}, 2}$.

The observations tell us the number of stars in well defined areas in the $l V$-diagrams. In our calculations we follow a star in an orbit defined by initial position $\boldsymbol{r}_{0}$ and an initial velocity $\dot{\boldsymbol{r}}_{0}$ and record the time that the star spends in each such area. We followed the orbit over a time long enough for the star to make several turns around the galactic centre. We checked that if we increased this time by a factor 2.5 (50000 time steps instead of 20000 ) the relative time spent in each area did not change significantly.

At a certain time $t$ all stars have the position $\left(r_{\text {rot }}, \phi_{\text {rot }}\right)$ in the rotating frame and the position $\left(r_{\text {in }}, \phi_{\text {in }}\right)$ in an inertial frame that coincided with the rotating frame at $t=0$. Because $r_{\text {in }}=r_{\text {rot }}$ and $\phi_{\text {in }}=\phi_{\text {rot }}+\Omega t$ the distribution of the stars in the rotating frame at time $t$ is equal to that in the inertial frame after rotating the former distribution over an angle $\Omega t$.

\subsection{The gravitational potential}

We adopt a simple potential taken from BT (their Eq. (3.77)):

$\Phi_{\text {bar }}(x, y)=\frac{1}{2} v_{0}^{2} \ln \left\{r_{0}^{2}+x^{2}+(1+\epsilon) y^{2}\right\}$.

The coordinate frame that underlies this potential rotates with an angular speed of $-60 \mathrm{~km} \mathrm{~s}^{-1} \mathrm{kpc}^{-1}$. When $\epsilon=0.0$ the potential is axially symmetric:

$\Phi_{\text {axisym }}(r, \phi)=\frac{1}{2} v_{\mathrm{o}}^{2} \ln \left\{r_{\mathrm{o}}^{2}+r^{2}\right\}$

where $r=\sqrt{x^{2}+y^{2}}$. A possible rotation of the frame must have no influence on the orbits of this axially symmetric potential. The calculations bear this out; in fact, we used this independence on $\Omega$ as one of the tests on our software.

For later use we define a circular velocity, $v_{\text {circ }}$, by using $v_{\text {circ }}{ }^{2}=(1 / r) \mathrm{d} \Phi / \mathrm{d} r$ which leads to $v_{\text {circ }}=v_{\mathrm{o}} r / \sqrt{r_{\mathrm{o}}^{2}+r^{2}}$.

Equation (5) is mathematically easy to use and it satisfies two important boundary conditions: (1) close to the galactic center the circular velocity increases linearly with $r$, as had been deduced from the narrow correlation between $l$ and $v_{\text {los }}$ : $v_{\mathrm{c}}=v_{\mathrm{o}}\left(r / r_{\mathrm{o}}\right) \mathrm{km} \mathrm{s}^{-1}$ (see above); (2) the circular velocity is effectively constant beyond $0.5 \mathrm{kpc}$. The correlation between $l$ and $v_{\text {los }}$ of $\mathrm{SiO}$ maser stars disappears for $|l| \geq 1^{\circ}$; therefore we will use $r_{\mathrm{o}}=150 \mathrm{pc}\left(=r_{\odot} \sin 1^{\circ}\right)$ and $v_{\mathrm{o}}=200 \mathrm{~km} \mathrm{~s}^{-1}$.

Equation (4) is also easy to handle. We chose $\epsilon=0.1$, a value of the same magnitude as that of the barred potential used by Bissantz et al. (2003) for their interpretation of the structure of the ISM in the inner MWG. This low value of $\epsilon$ guarantees that the bar has only a weak effect: in any point $(x, y)$ the gravitational accelerations in the $x$ - and $y$-directions, $a_{x}$ and $a_{y}$ respectively, have a ratio $a_{x} / a_{y} \geq 0.89 x / y$, i.e. only $11 \%$ lower than in the axially symmetric case. As a consequence, the rotating bar has a limited effect on the actual stellar orbits in general but may have much larger effects near a resonance. The value of $\epsilon$ is quite uncertain- see for some discussion Sect. 6.3.

\subsection{Orbital structure}

\subsubsection{Non-conservation of angular momentum}

The properties of the stellar orbits are best discussed in the polar coordinates, $(r, \phi)$, rather than in cartesian coordinates $(x, y)$. In polar coordinates the equations take the following form

$\Phi(r, \phi)=\frac{1}{2} v_{\mathrm{o}}^{2} \ln \left\{r_{\mathrm{o}}^{2}+r^{2}\left(1+\epsilon \sin ^{2} \phi\right)\right\}$

and

$\frac{\mathrm{d}^{2} r}{\mathrm{~d} t^{2}}=-\frac{v_{\mathrm{o}}^{2}}{r} \frac{1+\epsilon \sin ^{2} \phi}{r_{\mathrm{o}}^{2} / r^{2}+1+\epsilon \sin ^{2} \phi}+\frac{J^{2}}{r^{3}}$

and

$\frac{\mathrm{d} J}{\mathrm{~d} t}=-\frac{1}{2} v_{\mathrm{o}}^{2} \frac{\epsilon \sin 2 \phi}{r_{\mathrm{o}}^{2} / r^{2}+1+\epsilon \sin ^{2} \phi}$

where $J=r^{2}(\dot{\phi}+\Omega)$, i.e. $J$ is the angular momentum in the inertial frame.

Both potentials, $\Phi_{\text {bar }}$ and $\Phi_{\text {axisym }}$ are monotonously rising when $r$ increases and thus all orbits are bound. The first term in the right-hand side of Eq. (7) equals the gravitational attraction $(=\mathrm{d} \Phi / \mathrm{d} r)$ and the second term equals the centrifugal acceleration $\left(J^{2} / r^{3}\right)$. If $J$ has a lower limit different from 0 there will be a minimal radius, $r_{\min }$, where the centrifugal acceleration equals the gravitational acceleration and where the radial velocity, $v_{\text {rad }}$, equals zero.

Some more insight is found by rewriting Eq. (8) as follows:

$\dot{\phi} \frac{\mathrm{d} J}{\mathrm{~d} \phi}=-\frac{1}{2} v_{\mathrm{o}}^{2} \frac{\epsilon \sin 2 \phi}{r_{\mathrm{o}}^{2} / r^{2}+1+\frac{1}{2} \epsilon(1-\cos 2 \phi)}$.

The right hand side is independent of $r$ when $r \gg r_{\mathrm{o}}$; say, when $r>0.5 \mathrm{kpc}$. Divide the $x y$-plane in four quadrants in the usual fashion. Integrating this equation over any of the quadrants, the integral will have the same absolute value as the integral over each of the other three; only the sign is different: over Q1 and Q3 the integral will be positive, over quadrants Q2 and Q4 the integral is negative. A star gains as much angular momentum in a given quadrant as it looses in the next; periodic orbits are possible. Only when large non-linear variations occur in $\dot{\phi}$ the star will gain or loose net angular momentum over one revolution.

\subsubsection{Co-rotation and outer and inner lindblad resonance}

The assumption of small variations in $J$ does no longer hold for those orbits that meet the bar each time under the same conditions: then the accumulation of effects due the weak force may be large. 

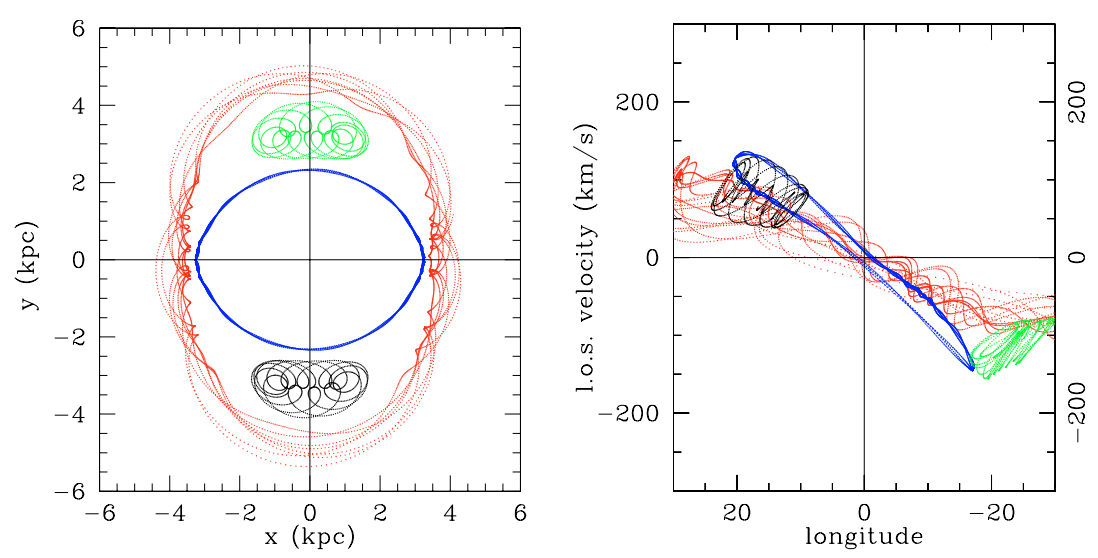

Fig. 4. Illustration of the co-rotation effect on almost circular orbits. The two orbits in red and in blue start at the center of the bar just inside and just outside co-rotation (at 3.25 and at $3.40 \mathrm{kpc}$ from the center) and with practically the same velocity in the $y$-direction $\left(-199.8 \mathrm{~km} \mathrm{~s}{ }^{-1}\right)$. When they cross the $y$-axis they are separated over a distance of about $1.2 \mathrm{kpc}$. The green and black orbits start on the $y$-axis at $3.33 \mathrm{kpc}$ and $-3.33 \mathrm{kpc}$ from the GC with a velocity of +179.8 and $-179.8 \mathrm{~km} \mathrm{~s}^{-1}$. The stars on either the green or the black orbit are practically stationary on the $x y$-plane and in the $l V$-diagram: see both diagrams. Stars on green and black orbits will cause a well-marked cluster of points in the stellar $l V$-diagram. Such clusters are not seen and thus the green and black orbits are not populated.

The first case is that of stars on a circular orbit with a velocity equal to that of the bar, i.e. when $v_{\text {circ }}=r \Omega$. This radius is called the radius of co-rotation, $r_{\mathrm{CR}}$; in our calculation $r_{\mathrm{CR}}=$ $200 / 60 \mathrm{kpc}=3.33 \mathrm{kpc}$. Such stars have zero velocity in the rotating frame, the gravitational attraction equals the centrifugal force and at first sight it looks as if the star will remain in the middle of the bar when that rotates around the GC. On closer look this circular orbit is unstable. Suppose that the star moves a little bit inward, into the direction of the GC, without changing its tangential velocity. Then the star will slowly move ahead of the bar; gravitational attraction by the bar will decrease the angular momentum and the gravitational attraction will pull the star farther inward. The opposite happens when the star receives a small velocity in the outward direction: now the bar will accelerate the star, the centrifugal force increases and pushes the star further outside. Thus orbits starting just outside of co-rotation and orbits starting just inside of co-rotation have the remarkable property that, while they originate from two neighboring points at almost the same velocity they separate over some distance when $x=0$. The red and blue orbits in Fig. 4 illustrate this.

The area around the $y$-axis between the two orbits can be filled by high angular-momentum orbits. See the green and black orbits in Fig. 4 that start at the $y$-axis at $y= \pm 3.3 \mathrm{kpc}$ with almost circular velocity in the tangential direction and zero velocity in the radial direction. These orbits are almost stationary in the rotating frame and thus also in the $l V$-diagram; the two locations in the $x y$-plane are called "Lagrangian points" (BT). If the population of these orbits is significant one should see a cluster of points in the $l V$-diagram: one at $l=-25^{\circ}$ and $v_{\text {los }}=-116 \mathrm{~km} \mathrm{~s}^{-1}$ and the other at $l=+17^{\circ}$ and $v_{\text {los }}=+78 \mathrm{~km} \mathrm{~s}^{-1}$. Such a cluster of sources is not seen in the two observed stellar $l V$-diagram diagrams and we must assume that the orbits around the Lagrangian points are populated by at most a few stars if at all. As a consequence one expect voids, areas without stars, between the two orbits; the voids have the shape of the crescent of the Moonagain: see Fig. 4.

Another consequence is that the two points at $(x, y)=$ $\left( \pm r_{\mathrm{CR}}, 0\right)$ are points of contact between two regions that are otherwise separated Through these points a flow of ISM can be established, inward or outward. Later we will come back to this point.
The second resonance is found for high-angular momentum orbits at a radius of $5.0 \mathrm{kpc}$ and this is $3 / 2$ times the co-rotation radius. This shows that we encounter there the Outer Lindblad Resonance (=OLR). Its effects are mild. Orbits close to the OLR have a larger extent in the $y$-direction than in the $x$-direction.

A third resonance, the Inner Lindblad Resonance (=ILR), is found at $r=0.9 \mathrm{kpc}$. It is caused by a resonance between the epicyclic frequency and the period of rotation around the GC. The ILR is irrelevant for our models, because in that region of the Milky Way we use only orbits of small angular momentum and these are not affected by the ILR.

\subsubsection{Low angular momentum orbits within $1 \mathrm{kpc}$ from the GC: rosette and bar orbits}

Low angular-momentum orbits within about $1 \mathrm{kpc}$ from the GC turn out to have an interesting property. The orbits have the shape made by a succession of thin loops. Each loop is roughly symmetric with respect to the GC; its very elongated shape (an axial ratio of 10 to 1 is typical) is the result of an interplay between the gravitational attraction and the Coriolis force; the centrifugal force turns out to be unimportant. As expected, the bar has a minor effect on the shape and size of the loop: compare the orbits (a) and (b) in Fig. 5 that start at the same point, but while orbit (a) is calculated for the axially symmetric potential $(\epsilon=0)$, orbit (b) is calculated for the barred potential $(\epsilon=0.11)$. Orbits (b) and (c) have been calculated for the same potential and they start at the same distance from the GC but (b) starts on the $y$-axis and (c) on the $x$-axis.

The bar-potential thus produces an interesting secular effect. Whereas orbit (b) describes a rosette around the GC, orbit (c) is restrained in the $y$-direction; its azimuth does not rotate around the GC. The star describes a rectangle: orbits starting inside the bar remain inside the bar. Orbits similar to orbit (c) will be called "bar orbits", orbits like orbit (b) will be called "rosette orbits". We attribute the difference between bar and rosette orbits to the gravitational attraction by the bar.

Bar and rosette orbits project differently in the $l V$-diagramsee the four diagrams at the top of Fig. 6. The rosette orbits cut the line-of-sight at a large variety of angles and $v_{\text {los }}$ has a large range of values. Stars on bar orbits travel up and down 

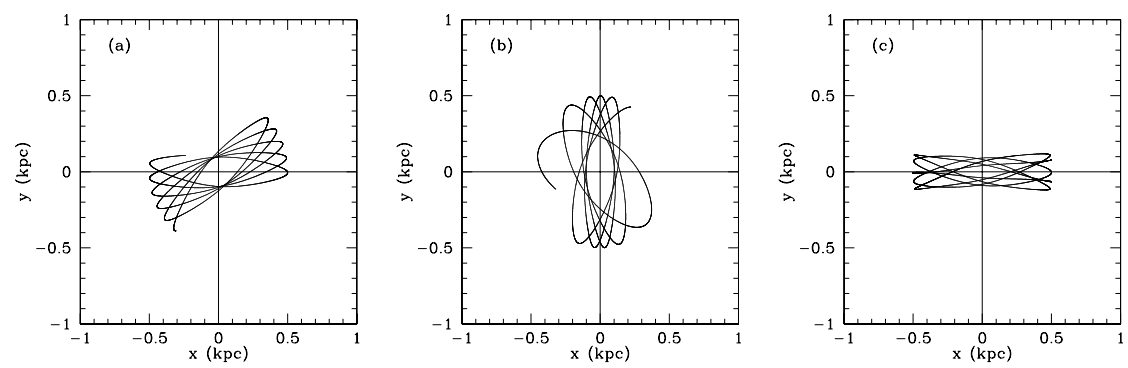

Fig. 5. Three comparable orbits. Orbit (a) has been calculated in the axially symmetric potential $(\epsilon=0.0)$ and started at a distance of $0.5 \mathrm{kpc}$ from the GC with an initial radial velocity $v_{\text {rad }}=0$ and a tangential velocity $v_{\text {tang }}=57.5 \mathrm{~km} \mathrm{~s}^{-1}$. Orbits (b) and (c) have been calculated for the bar potential using $\epsilon=0.11$. Orbit (b) started at the $y$-axis with $v_{\text {rad }}=0$ and $v_{\text {tang }}=+57.5 \mathrm{~km} \mathrm{~s}^{-1}$ and orbit (c) started at the $x$-axis at $x=0.5 \mathrm{kpc}$ and an initial velocity $v_{\text {rad }}=0$ and $v_{\text {tang }}=-57.5 \mathrm{~km} \mathrm{~s}^{-1}$. For clarity we show only a small part of the orbit. Orbits of type c are called "rosette-orbits", orbits of type d are called "bar-orbits".
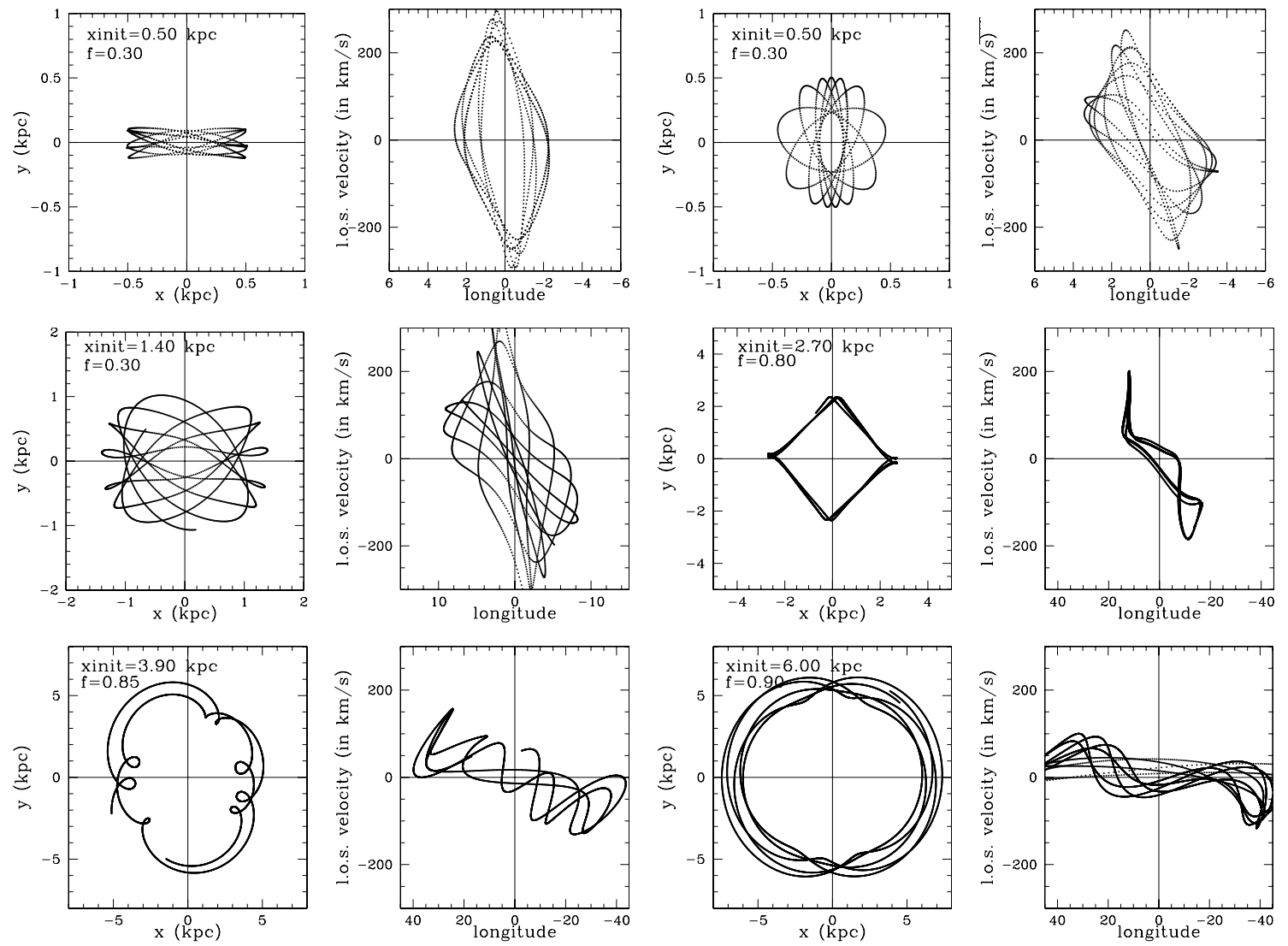

Fig. 6. Six pairs of diagrams, each pair referring to a specific orbit. Each diagram contains $2000 * x_{\text {init }}$ stars $\left(x_{\text {init }}\right.$ in kpc) that started their journey in the bar at a distance $x_{\text {init }}$ from the center under identical initial conditions but at successive times separated by $2.5 \mathrm{Mjr}$. In each diagram the numbers in the upper left corner specify the orbit (see text). Of each pair of diagrams the one on the left is the distribution in the galactic plane and the diagram at the right-hand side is its projection onto the $l V$-diagram. The horizontal scale varies from one $l V$-diagram to the next.

the bar and the line-of-sight always cuts the orbits at very similar angles; consequentially one finds high values for $v_{\text {los }}$ more often and they distribute more evenly among permitted and forbidden quadrants. This is illustrated in Table 1 and discussed in Sect. 5.5. Orbits \#3 and \#8 are the same as orbits in Fig. 5b and in Fig. 5c. Whereas orbit 8 spends essentially zero time in area 7 , orbit 3 spends $10 \%$ of its time there.

\subsection{Orbit library}

The orbits in $\Phi_{\text {bar }}$ are characterized by the initial values for $x$, $y, v_{x}$ and $v_{y}$. To obtain a complete library of orbits one needs to explore a four-dimensional space. This is a huge task and there is the risk of using "twin orbits": two orbits that set out on different initial conditions but one of the two passes through the starting point of the other with the same velocity, so that the two orbits are the same. To obtain an orbit library representative for our sample we used a selection method that works very well for orbits belonging to $\Phi_{\text {axisym }}$ : label orbits by their initial distance, $r_{\text {init }}$, and adopt $v_{\text {rad }}=0$ and $v_{\text {tang }}=f v_{\text {circ }}$, where $f$ is a constant with values between 0 and 1 . By chosing $v_{\text {rad }}=0$ one ensures that $r_{\text {init }}$ is the apocenter of the orbit.

To select orbits in $\Phi_{\text {bar }}$ we chose various values for $r_{\text {init }}$ and for $f$. For each pair $\left(r_{\text {init }}, f\right)$ we started one orbit at $(x, y)=$ $\left(r_{\text {init }}, 0\right)$ with velocity $\left(0,-f v_{\text {circ }}\right)$ and a second at $(x, y)=\left(0, r_{\text {init }}\right)$ with a velocity $\left(f v_{\text {circ }}, 0\right)$; we used values for $r_{\text {init }}$ between 0.50 to $7.50 \mathrm{kpc}$, and values for $f=0.3,0.5,0.85,1.0$. 
Table 1. $\%$ of time spent in each selected area.

\begin{tabular}{rr|rrrrrrrr}
\hline \hline gr. & orb. & 1 & 2 & 3 & 4 & 5 & 6 & 7 & out \\
\hline 1 & 3 & 0 & 0 & 19 & 40 & 0 & 31 & 10 & 0 \\
2 & 8 & 0 & 0 & 8 & 71 & 0 & 21 & 0 & 0 \\
3 & 16 & 3 & 23 & 15 & 43 & 1 & 14 & 2 & 0 \\
4 & 23 & 14 & 69 & 0 & 8 & 1 & 8 & 0 & 0 \\
5 & 29 & 55 & 16 & 0 & 8 & 12 & 6 & 0 & 2 \\
6 & 38 & 51 & 2 & 0 & 3 & 22 & 4 & 0 & 18 \\
\hline
\end{tabular}

\subsection{The projection of orbits on the areas in the IV-diagrams}

The two upper $l V$-diagrams of Fig. 3 contain all our information on the orbits of the maser stars. The importance of each dot in the diagrams depends on where it is found and precisely this is the idea behind our definition of the boundaries of the seven areas (as given in the diagram on the lower right in Fig. 3): a priori we judge each area to be of the same importance. For example, area 7 contains only 12 stars and yet these stars are essential because the orbits on which they move are not implied by the dots in any of the other areas. In contrast, area 1 contains 355 stars that together represent all orbits of high angular momentum outside of the molecular ring, i.e. the population of the galactic disk.

Table 1 illustrates this quantitatively. Each row refers to an orbit that is characteristic for its group. The full specification of each orbit is given in Table 2, see farther below. Table 1 gives the percentage of time of each orbit spends in each area (Cols. 3 to 9). Column 10 contains the fraction of time spent outside the longitude range considered, i.e. the time spent at $|l| \geq 45^{\circ}$. It is easily seen that only stars on orbits of group 1 and 3 populate area 7.

\subsection{The comparison of observations and models; $\chi^{2}$-values}

We selected orbits from the orbit library and labelled each with the suffix $i$. Each orbit defines a probability distribution in the $l V$ diagram, $p_{i}(l, V)$. We calculated the integral of over each critical area $j$ and thus predicted the number of stars, $N_{\text {pred,j }}$, in each area, $j$ :

$N_{\text {pred }, \mathrm{j}}=N_{\text {total }} \Sigma_{i} w_{i} \iint_{j} p_{i}(l, V) \mathrm{d} l \mathrm{~d} V$

where we introduced weights $w_{i}$ that will be assigned to each orbit. The weight is thus proportional to the number of stars on this orbit. Later we determined a multiplication factor $W=$ $771 / \Sigma_{i} w_{i} ; 771$ is the total number of $\mathrm{OH} / \mathrm{IR}$ stars of our sample. After starting the calculations it quickly turned out that 44 individual orbits is a too large number too handle conveniently and we formed six groups of orbits and give the same weight $w_{\mathrm{i}}$ and the same value of $f$ to all members of a group.

We compared the numbers observed in each area, $N_{\text {obs }}$, with the numbers predicted, $N_{\text {pred }}$. We assume that the probability distribution of $N_{\text {obs }}$ is Poisson-like and thus that the dispersion in the observed numbers equals the mean value. We therefore use the two statistics

$\chi_{1}^{2}=\frac{\left(N_{\text {obs }}^{\mathrm{nm}}-N_{\text {pred }}^{\mathrm{nm}}\right)^{2}}{N_{\text {pred }}^{\mathrm{nm}}}$

and

$\chi_{2}^{2}=\frac{\left(N_{\mathrm{obs}}^{\mathrm{nm}}-N_{\mathrm{pred}}^{\mathrm{nm}}\right)^{2}}{N_{\mathrm{obs}}^{\mathrm{nm}}}$.
Table 2. Number of stars in each of the seven areas.

\begin{tabular}{r|ccccccc|c}
\hline \hline area: & 1 & 2 & 3 & 4 & 5 & 6 & 7 & sum \\
\hline pred. & 336 & 104 & 28 & 123 & 88 & 82 & 12 & 773 \\
obs. & 355 & 108 & 19 & 104 & 92 & 82 & 11 & 771 \\
\hline$\chi_{1}^{2}$ & 1.1 & 0.2 & 2.9 & 2.9 & 0.2 & 0.0 & 0.1 & 7.3 \\
$\chi_{2}^{2}$ & 1.0 & 0.1 & 4.3 & 3.5 & 0.2 & 0.0 & 0.1 & 9.2 \\
\hline
\end{tabular}

Ideally one expects the values of $\chi^{2}$ to be 1 . In our case, when comparing observations with predictions, we adjust the values of $w_{i}$ to minimize $\left\langle\chi^{2}\right\rangle$.

\section{Results and discussion}

After some experimentation we decided to use 44 orbits arranged in six groups. Each orbit is defined by an initial position and an initial velocity- see Table 3 that contains information on all orbits ("orb.") used, on the group ("gr") to which they were associated and on the weights (" $w_{i}$ ") that we finally assigned.

Figure 6 contains six pairs of diagrams representing the six groups of stars; for clarity they contain only about $10 \%$ of the points that have been calculated. The left diagram of each pair shows the orbit projected on the galactic plane. The diagram on the right gives the distribution in the $l V$-diagram.

\subsection{Choice of weights that produce the best fit to the observations}

We start by filling area 7 that contains $11 \mathrm{OH} / \mathrm{IR}$ stars with high velocities $\left(\left|v_{\text {los }}\right|>150 \mathrm{~km} \mathrm{~s}^{-1}\right)$ in the forbidden quadrants Q2 and Q4. It is easily seen in Table 1 that stars of group 1 are especially needed for this purpose; these are the stars on bar orbits as discussed above. These stars will also begin to fill areas 3, 4 and 6. Next consider areas 1 and 2: these will be filled predominantly by stars on the orbits of groups 5 and 6 . The remaining areas $(3,4,5,6)$ have now been partially filled by orbits of groups 1, 5 and 6 and we use the other groups to complete the population of the remaining areas. This led us to a final assignment of the weights $w_{i}$ as given in Table 3 . This table contains a specification of the solution with the smallest value of $\Sigma \chi_{i}^{2}$. The first column contains the number of the orbit and in Cols. (2) through (5) the orbit is specified. Columns (6) through (13) contain the number of maser stars in each of the seven counting areas and their sum. Column (14) gives the number of stars on this orbit but outside of the range, i.e stars for which $|l|>45^{\circ}$.

Using the orbits and weights specified in Table 3 we find the total number of stars in each of the seven areas; see Table 2. The first row in Table 2 gives the total number of stars predicted, the second row gives the number as observed. In rows three and four we show for each area the two $\chi^{2}$-values that follow from the comparison of predictions and observations (see above).

\subsection{The distribution of maser stars in the Inner MWG}

An important result of our simulation are the best weights, $w_{i}$, that we assigned to each group of orbits. This set of weights and the properties of each orbit represent together the distribution function as known from statistical mechanics. We may use this function to calculate the spatial distribution of the stars. The left diagram in Fig. 7 shows the result obtained after smoothing the somewhat noisy image. The map shows two striking features. First there is the rectangular shaped distribution of stars near the GC: the galactic bar. The existence of the bar is required by the 
Table 3. All orbits, their parameters and their contribution to the 7 areas.

\begin{tabular}{|c|c|c|c|c|c|c|c|c|c|c|c|c|c|}
\hline \multirow[t]{2}{*}{ orb } & \multirow{2}{*}{$\begin{array}{l}x_{\text {init }} \\
\mathrm{kpc}\end{array}$} & \multirow{2}{*}{$\begin{array}{l}y_{\text {init }} \\
\mathrm{kpc}\end{array}$} & \multirow{2}{*}{$\begin{array}{c}v_{x, \text { init }} \\
\mathrm{km} \mathrm{s}^{-1}\end{array}$} & \multirow{2}{*}{$\begin{array}{c}v_{y, \text { init }} \\
\mathrm{km} \mathrm{s}^{-1}\end{array}$} & \multicolumn{7}{|c|}{ predicted number in each area } & \multirow[t]{2}{*}{ sum } & \multirow[t]{2}{*}{ outside } \\
\hline & & & & & [1] & [2] & [3] & [4] & {$[5]$} & [6] & [7] & & \\
\hline (1) & (2) & (3) & (4) & (5) & (6) & (7) & (8) & (9) & (10) & (11) & (12) & 913) & (14) \\
\hline \multicolumn{14}{|c|}{ group $1, f_{1}=0.30, w_{1}=2.00$} \\
\hline 1 & 0.30 & 0.00 & 0 & -54 & 0 & 0 & 3 & 11 & 0 & 7 & 2 & 23 & 0 \\
\hline 2 & 0.40 & 0.00 & 0 & -56 & 0 & 0 & 4 & 9 & 0 & 7 & 2 & 22 & 0 \\
\hline 3 & 0.50 & 0.00 & 0 & -57 & 0 & 0 & 4 & 9 & 0 & 7 & 2 & 22 & 0 \\
\hline 4 & 0.60 & 0.00 & 0 & -58 & 0 & 0 & 4 & 9 & 0 & 7 & 2 & 22 & 0 \\
\hline 5 & 0.70 & 0.00 & 0 & -59 & 0 & 0 & 5 & 8 & 0 & 7 & 2 & 22 & 0 \\
\hline 6 & 0.80 & 0.00 & 0 & -59 & 0 & 0 & 5 & 8 & 0 & 7 & 2 & 22 & 0 \\
\hline \multicolumn{14}{|c|}{ group $2, f_{2}=0.30, w_{2}=0.10$} \\
\hline 7 & 0.00 & 0.30 & 54 & 0 & 0 & 0 & 0 & 1 & 0 & 0 & 0 & 1 & 0 \\
\hline 8 & 0.00 & 0.50 & 57 & 0 & 0 & 0 & 0 & 1 & 0 & 0 & 0 & 1 & 0 \\
\hline 9 & 0.00 & 0.70 & 59 & 0 & 0 & 0 & 0 & 1 & 0 & 0 & 0 & 1 & 0 \\
\hline 10 & 0.00 & 0.80 & 59 & 0 & 0 & 0 & 0 & 1 & 0 & 0 & 0 & 1 & 0 \\
\hline 11 & 0.00 & 0.90 & 59 & 0 & 0 & 0 & 0 & 1 & 0 & 0 & 0 & 1 & 0 \\
\hline \multicolumn{14}{|c|}{ group $3, f_{3}=0.30, w_{3}=0.20$} \\
\hline 12 & 1.00 & 0.00 & 0 & -59 & 0 & 0 & 0 & 1 & 0 & 1 & 0 & 2 & 0 \\
\hline 13 & 1.10 & 0.00 & 0 & -59 & 0 & 0 & 1 & 1 & 0 & 1 & 0 & 3 & 0 \\
\hline 14 & 1.20 & 0.00 & 0 & -60 & 0 & 0 & 1 & 1 & 0 & 1 & 0 & 3 & 0 \\
\hline 15 & 1.30 & 0.00 & 0 & -60 & 0 & 0 & 1 & 1 & 0 & 1 & 0 & 3 & 0 \\
\hline 16 & 1.40 & 0.00 & 0 & -60 & 0 & 1 & 0 & 1 & 0 & 0 & 0 & 2 & 0 \\
\hline 17 & 1.50 & 0.00 & 0 & -60 & 0 & 1 & 0 & 1 & 0 & 0 & 0 & 2 & 0 \\
\hline 18 & 1.70 & 0.00 & 0 & -60 & 0 & 1 & 0 & 1 & 0 & 0 & 0 & 2 & 0 \\
\hline 19 & 1.90 & 0.00 & 0 & -60 & 0 & 0 & 0 & 0 & 0 & 0 & 0 & 0 & 0 \\
\hline \multicolumn{14}{|c|}{ group $4, f_{4}=0.80, w_{4}=0.95$} \\
\hline 20 & 2.10 & 0.00 & 0 & -160 & 0 & 7 & 0 & 3 & 0 & 1 & 0 & 11 & 0 \\
\hline 21 & 2.30 & 0.00 & 0 & -160 & 0 & 7 & 0 & 3 & 0 & 1 & 0 & 11 & 0 \\
\hline 22 & 2.50 & 0.00 & 0 & -160 & 0 & 7 & 0 & 2 & 0 & 1 & 0 & 10 & 0 \\
\hline 23 & 2.70 & 0.00 & 0 & -160 & 2 & 7 & 0 & 1 & 0 & 1 & 0 & 11 & 0 \\
\hline 24 & 2.90 & 0.00 & 0 & -160 & 1 & 6 & 0 & 2 & 0 & 1 & 0 & 10 & 0 \\
\hline 25 & 3.10 & 0.00 & 0 & -160 & 2 & 6 & 0 & 2 & 0 & 1 & 0 & 11 & 0 \\
\hline 26 & 3.30 & 0.00 & 0 & -160 & 3 & 5 & 0 & 2 & 0 & 1 & 0 & 11 & 0 \\
\hline \multicolumn{14}{|c|}{ group $5, f_{5}=0.85, w_{5}=2.00$} \\
\hline 27 & 3.50 & 0.00 & 0 & -170 & 6 & 11 & 0 & 4 & 0 & 1 & 0 & 22 & 0 \\
\hline 28 & 3.70 & 0.00 & 0 & -170 & 7 & 10 & 0 & 3 & 0 & 2 & 0 & 22 & 0 \\
\hline 29 & 3.90 & 0.00 & 0 & -170 & 12 & 4 & 0 & 2 & 3 & 1 & 0 & 22 & 2 \\
\hline 30 & 4.10 & 0.00 & 0 & -170 & 13 & 5 & 0 & 2 & 2 & 1 & 0 & 23 & 1 \\
\hline 31 & 4.30 & 0.00 & 0 & -170 & 15 & 4 & 0 & 2 & 0 & 1 & 0 & 22 & 0 \\
\hline 32 & 4.50 & 0.00 & 0 & -170 & 15 & 3 & 0 & 3 & 0 & 1 & 0 & 22 & 0 \\
\hline \multicolumn{14}{|c|}{ group $6, f_{6}=0.85, w_{6}=3.75$} \\
\hline 33 & 4.75 & 0.00 & 0 & -170 & 24 & 5 & 0 & 4 & 5 & 2 & 0 & 40 & 3 \\
\hline 34 & 5.00 & 0.00 & 0 & -170 & 26 & 5 & 0 & 4 & 3 & 3 & 0 & 41 & 3 \\
\hline 35 & 5.25 & 0.00 & 0 & -170 & 16 & 4 & 0 & 4 & 7 & 3 & 0 & 34 & 20 \\
\hline 36 & 5.50 & 0.00 & 0 & -170 & 18 & 2 & 0 & 3 & 7 & 1 & 0 & 31 & 24 \\
\hline 37 & 5.75 & 0.00 & 0 & -170 & 20 & 2 & 0 & 1 & 8 & 2 & 0 & 33 & 20 \\
\hline 38 & 6.00 & 0.00 & 0 & -170 & 21 & 1 & 0 & 1 & 9 & 2 & 0 & 34 & 18 \\
\hline 39 & 6.25 & 0.00 & 0 & -170 & 23 & 0 & 0 & 1 & 9 & 1 & 0 & 34 & 16 \\
\hline 40 & 6.50 & 0.00 & 0 & -170 & 24 & 0 & 0 & 1 & 9 & 1 & 0 & 35 & 15 \\
\hline 41 & 6.75 & 0.00 & 0 & -170 & 24 & 0 & 0 & 1 & 8 & 1 & 0 & 34 & 16 \\
\hline 42 & 7.00 & 0.00 & 0 & -170 & 24 & 0 & 0 & 2 & 6 & 2 & 0 & 34 & 21 \\
\hline 43 & 7.25 & 0.00 & 0 & -170 & 21 & 0 & 0 & 2 & 6 & 2 & 0 & 31 & 27 \\
\hline 44 & 7.50 & 0.00 & 0 & -170 & 19 & 0 & 0 & 2 & 6 & 2 & 0 & 29 & 33 \\
\hline
\end{tabular}

stars in area 7 (high velocities near the GC and in the forbidden quadrants). The $30 \mathrm{OH} / \mathrm{IR}$ stars in areas 3 and 7 belong to the bar; they travel up and down the bar. The second remarkable feature is the presence of two empty regions, the voids, one with the shape of the crescent of the Moon at first quarter, the other with that of the Moon at last quarter. The existence of these voids is described in Sect. 5.3.2; there it is also argued that the observations show the absence of stars in these crescents; the crescents are voids in the maser star distribution. The relative size of the voids is determined by the strength of the bar, i.e. by the value of the parameter $\epsilon$ in Eq. (4). The voids disappear when $\epsilon \rightarrow 0$.
We point out that our value for co-rotation, $3.3 \mathrm{kpc}$ agrees with the radius of the molecular ring. It is likely that there is a relation between the presence of the bar inside $3.3 \mathrm{kpc}$ and the (almost) complete absence of ISM inside of this distance- with the exception of the nuclear disk in the immediate neighbourhood of SgrA; below we suggest a mechanism that may cause a rapid transport of ISM from the molecular ring to the very center of the MWG.

The structure shown in Fig. 7 is similar to the figure derived by one of us in an earlier paper (Sevenster 1999b). She derived her conclusions on very different arguments, namely on a 

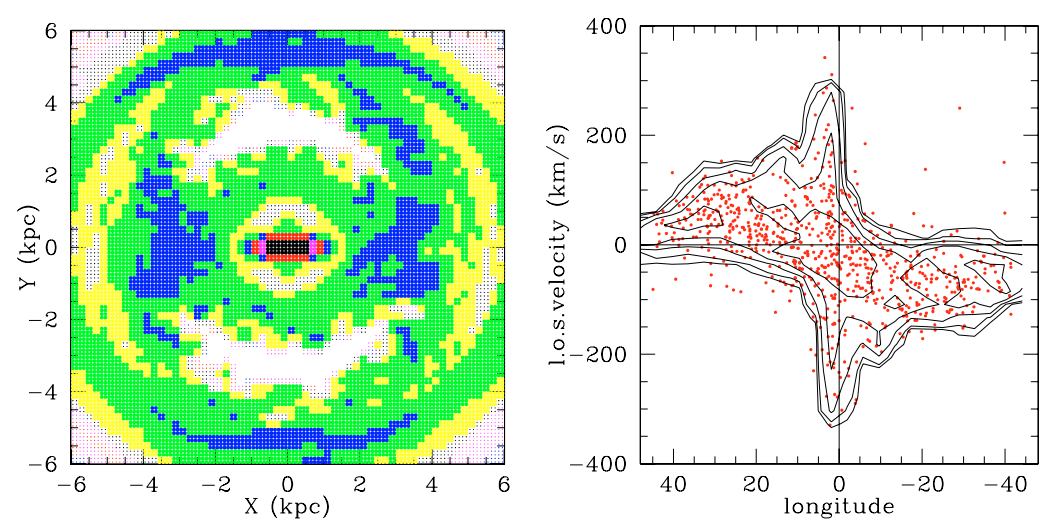

Fig. 7. On the left our ultimate contour diagram showing the reconstructed distribution of the maser stars in an area of $12 \times 12 \mathrm{kpc} \mathrm{c}^{2}$ centered on the GC. Notice the bar like distribution near the center and the voids at about $(x, y)=(0.0 \mathrm{kpc}, \pm 3 \mathrm{kpc})$. The average stellar density can be read off from the colors: black: 110 maser stars per $\mathrm{kpc}^{2}$, magenta: 56; red: 24, blue 8.9, green 5.4, yellow 2.6; other colors indicate less than 1.0 star per $\mathrm{kpc}^{2}$; the white area does not contain a single star. On the right the corresponding diagram of longitude versus line-of-sight-velocity of the $\mathrm{OH} / \mathrm{IR}$ stars. The contours in black illustrate our prediction and the red dots represent the observed distribution of the OH/IR stars.

morphological study of the distribution of the stars in a diagram of longitude versus latitude.

\subsection{Is our representation of the IV-diagrams by orbits unique?}

We have obtained a set of orbits that fit the observations quite well and this led us to the reconstruction of the distribution of maser stars shown in Fig. 7. There remains at least one important question: can other sets be found that predict $l V$-diagrams equally well and yet give a very different distribution of the maser stars in the galactic plane?

We started from a large library of orbits from which we selected a limited group of orbits that might be significant. We concluded earlier that outside of the radius of the molecular ring, 3 to $3.5 \mathrm{kpc}$, the orbits have high angular-momentum as is appropriate for the galactic disk. Inside $3 \mathrm{kpc}$ the velocity distribution changes, as the orbits become more and more elongated. These considerations are reflected in the list of orbits shown in Table 3. Once this selection of orbits had been made the assignment of the weights followed more or less straightforwardly. We thus do not expect that a qualitatively very different solution can be found with the same low value of $\Sigma \chi_{i}^{2}$.

Consider now the values of the two most uncertain free parameters, $\epsilon$, the strength of the bar-like part of our gravitational potential, and $\phi_{\odot}$, the angle between the Sun-GC line and the bar. We adopted $\epsilon=0.11$, partially on intuition and partially because it is in the same range as the potential used by Bissantz et al. in their analysis of the ISM distribution in the inner MWG. We redid our analysis assuming $\epsilon=0.05$ and found a set of weights by which $\Sigma \chi_{i}^{2}=10$ but the overall shape of the distribution looked suspect. We concluded that a value of $\epsilon=0.05$ is barely acceptable and that a lower value than 0.05 is ruled out. Taking $\epsilon=0.2$ led to predicted $l V$-diagrams that contained too many sources in area 7. Our estimate is thus that $\epsilon$ has a value between 0.05 and 0.2 , but the uncertainty in this value we cannot specify quantitatively. With respect to $\phi_{\odot}$ we have experimented with values of $20^{\circ}$ and $40^{\circ}$ and find easily equally satisfying solutions. From star count data in the mid-infrared taken with the SPITZER satellite Benjamin et al. (2005) find a value of $\phi_{\odot}=45^{\circ}$.

In this paper our approach has been to chose a galactic potential that contains a rotating bar, followed by orbit calculations and then by a selection of those orbits that predict as well as possible the observed $l V$-diagrams. Given this approach we think that the end result is the best possible, and actually that it is quite good. There are however enough uncertainties in our approach (how reliable is the potential; are there many stars below the detection limits of the maser surveys and are the observed $l V$-diagrams sufficiently representative of the true distribution?) to leave some lingering doubts- even among the authors of this paper.

\subsection{Speculation about the " $3 \mathrm{kpc}$ arm"}

In the quite successful interpretation of the galactic survey of the $21 \mathrm{~cm}$ line in the 1950's there remained one major puzzle, an easily recognized feature that has never been properly explained. This is the so-called " $3 \mathrm{kpc}-\mathrm{arm}$ ", an object that is almost certainly not a spiral arm (i.e. it is not a ripple on the galactic distribution of the ISM) and may not be at $3 \mathrm{kpc}$ either. At $l=0^{\circ}$ it is seen as an absorption line against Sgr A with a line-of-sight-velocity of $-50 \mathrm{~km} \mathrm{~s}^{-1}$; hence it must lie between the Sun and the GC. This high line-of-sight velocity has never been explained satisfactorily, although many attempts have been made. There may be a counterpart to the $3 \mathrm{kpc}$-arm at the other side of the GC; it is seen as an elongated feature in $\mathrm{HI}$ and $\mathrm{CO}$ $l V$-diagrams with a line-of-sight-velocity of $+135 \mathrm{~km} \mathrm{~s}^{-1}$.

Sevenster (1999) suggested that the arm has its origin near one of the two points where the bar meets its corotation radius. Matter might "leak" through this and then fall inwards. In this model the $3 \mathrm{kpc}$ arm is a flow of matter from this corotation point inward that feeds gas to the clouds close to the GC, e.g. such clouds as Sgr B2.

We calculated orbits for stars. Clouds of ISM will follow the same orbits until they collide with other clouds. Extended structures of the ISM will face another problem: during a ballistic orbit some parts of the structure will be forced by the gravitational force to contract faster than the speed of sound and shocks will appear that may convert the kinetic energy of large-scale motions into thermal energy, i.e. into heat. We have checked this and find that in most of our ballistic orbits such rapid internal motions of 5 to $10 \mathrm{~km} \mathrm{~s}^{-1}$ occur during short periods. We suggest that the shock waves in the ISM and the consequential lowering of the kinetic energy of the gas may give the explanation for the low density of the ISM inside CR.

This conclusion led us to the following simulation. The points where the bar crosses the co-rotation circle will be named B1 and B2, with B1 being (at present) closer to the Sun than B2. 


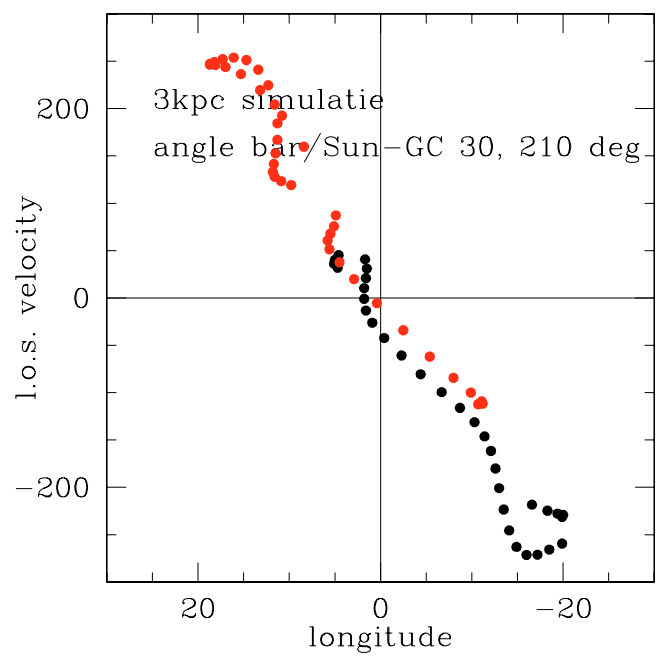

Fig. 8. The relation between longitude and $v_{\text {los }}$ for projectiles launched from the points of contact B1 (black symbols) and B2 (red symbols). We observe $l$ and $v_{\text {los }}$ of each projectile when the bar makes an angle of $30^{\circ}$ or of $210^{\circ}$ with the Sun-GC line. The time between two launches is approximately $5 \mathrm{Myr}$.

At successive times, separated by a fixed interval $\Delta t$, a projectile, i.e. a cloud of ISM or a star, is ejected from B1 or B2 into the inner MWG with an initial velocity $\dot{\boldsymbol{r}}_{\mathbf{0}}$. We follow all projectiles along their ballistic orbits in the same way as we followed the stars. When the clouds/projectile have completed half a revolution around the GC they dissolve. When the Sun and the bar reach their present mutual position we calculate the longitude and $v_{\text {los }}$ of all objects.

Figure 8 shows the velocities and longitudes of the "projectiles" shot from contact point B1 (black) or B2 (red) and observed from the Sun when the angle between the Sun-GC line and the central line of the bar equals $30^{\circ}$ or of $210^{\circ}$. This is a crude result: more simulations are needed but we think that our approach is promising and that the $3 \mathrm{kpc}$ arm may well be a channel through which gas is transported from the molecular ring to the galactic center area. A gas dynamical study is required to further develop this suggested explanation of the $3 \mathrm{kpc}$-arm.

\subsection{Conclusions and a few concluding remarks}

The following conclusions from our study we find the most important:

1. A qualitative comparison of the $l V$-diagrams of the ISM and of the maser stars shows overall agreement: stars and gas have the same overall distributions in space and in velocity. Small but significant differences can be attributed to the fact that the gas is on dissipative orbits and the maser stars are not.

2. The shape of the velocity distribution of the maser stars changes when one moves inward along the galactic plane: outside $3.3 \mathrm{kpc}$ the distribution is peaked around the circular velocity; inside this, i.e. inside of the molecular ring, radial motions become increasingly important.

3. Qualitative comparison between the $l V$-diagrams of the ISM and of the maser stars leads to the demarcation of seven areas in the $l V$-diagrams.

4. Adopting a potential that is predominantly axially symmetric but contains a weak, rotating bar we find a set of orbits that explains the distribution of longitudes and line-of-sightvelocities.
5. Inside $2 \mathrm{kpc}$ from the $\mathrm{GC}$ the stars are on bar orbits.

6. We also find two crescent-like voids where no star is found. These voids are caused by the co-rotation resonance.

7. The distribution of the maser stars as derived in this paper compares remarkably well to that presented by Sevenster (1999), based on partially the same data but on a very different type of argument.

Some concluding remarks

1. The analysis in this paper is $2 \mathrm{D}$ and it is based on a simple galactic potential. One of us $(\mathrm{GvdV})$ has started a larger 3D analysis.

2. It is technically possible to measure the proper motions of the $\mathrm{SiO}$ maser stars by interferometry. This will be a great step forward..

3. The $11 \mathrm{OH} / \mathrm{IR}$ stars in area 7 of the $l V$-diagram are moving up and down the bar. Their age is between 1 and 5 Gyr and thus they set a limit on the time of formation of the bar.

Acknowledgements. M.M.'s research has been made possible by a Grant from NOVA, the Netherlands Research School in Astronomy. We thank the referee, Anders Winnberg, for his fair and critical comments.

\section{References}

Baud, B., Habing, H. J., O’Sullivan, J. D., Winnberg, A., \& Matthews, H. E. 1975, Nature, 258, 406

Baud, B., Habing, H. J., Matthews, H. E., \& Winnberg, A. 1981, A\&A, 95, 156 Benjamin, R. A., Churchwell, E., Babler, B. L., et al. 2005, ApJ, 630, L149 Binney, J., \& Merrifield, M. 1998, Galactic Astronomy (Princeton, New Jersey, 08540, USA: Princeton University Press)

Binney, J., \& Tremaine, S. 1987, Galactic Dynamics (Princeton, New Jersey, 08540, USA: Princeton University Press)

Bissantz, N., Englmaier, P., \& Gerhard, O. 2003, MNRAS, 340, 949

Blitz, L., \& Spergel, D. N. 1991, ApJ, 379, 631

Bowers, P. F. 1978a, A\&A, 64, 307

Bowers, P. F. 1978b, A\&AS, 31, 127

Dame, T. M., Hartmann, D., \& Thaddeus, P. 2001, ApJ, 547, 792

Debattista, V. P., Gerhard, O., \& Sevenster, M. N. 2002, MNRAS, 334, 355

Deguchi, S., Fujii, T., Glass, I. S., et al. 2004, PASJ, 56, 765

Dwek, E., Arendt, R. G., Hauser, M. G., et al. 1995, ApJ, 445, 716

Elldér, J., Rönnäng, B., \& Winnberg, A. 1969, Nature, 222, 67

Habing, H., \& Olofsson, H. 2003, Asymptotic Giant Branch Stars (New York, USA: Springer)

Kaifu, N., Buhl, D., \& Snyder, L. E. 1975, ApJ, 195, 359

Knowles, S. H., Mayer, C. H., Cheung, A. C., Rank, D. M., \& Townes, C. H. 1969, Science, 163, 1055

Lindqvist, M., Habing, H. J., \& Winnberg, A. 1992a, A\&A, 259, 118

Lindqvist, M., Winnberg, A., Habing, H. J., \& Matthews, H. E. 1992b, A\&AS, 92, 43

Messineo, M., Habing, H. J., Sjouwerman, L. O., Omont, A., \& Menten, K. M. 2002, A\&A, 393, 115

Messineo, M., Habing, H. J., Menten, K. M., Omont, A., \& Sjouwerman, L. O. 2004, A\&A, 418, 103

Messineo, M., Habing, H. J., Menten, K. M., et al. 2005, A\&A, 435, 575

Sevenster, M. N. 1999, MNRAS, 310, 629

Sevenster, M. N., Chapman, J. M., Habing, H. J., Killeen, N. E. B., \& Lindqvist, M. $1997 \mathrm{a}$, A\&AS, 122,79

Sevenster, M. N., Chapman, J. M., Habing, H. J., Killeen, N. E. B., \& Lindqvist, M. 1997b, A\&AS, 124, 509

Sevenster, M., Saha, P., Valls-Gabaud, D., \& Fux, R. 1999, MNRAS, 307, 584

Sevenster, M. N., Dejonghe, H., Van Caelenberg, K., \& Habing, H. J. 2000, A\&A, 355, 537

Sevenster, M. N., van Langevelde, H. J., Moody, R. A., et al. 2001, A\&A, 366, 481

Sjouwerman, L. O., van Langevelde, H. J., Winnberg, A., \& Habing, H. J. 1998, A\&AS, 128, 35

Te Lintel Hekkert, P., Caswell, J. L., Habing, H. J., et al. 1991, A\&AS, 90, 327

Tremaine, S., \& Weinberg, M. D. 1984, ApJ, 282, L5

Weiland, J. L., Arendt, R. G., Berriman, G. B., et al. 1994, ApJ, 425, L81

Wilson, W., \& Barrett, A. 1968, AJ, 73, 209

Winnberg, A. 2003, in Mass-Losing Pulsating Stars and their Circumstellar Matter, ed. Y. Nakada, M. Honma, \& M. Seki, ASSL, 283, 367 